\title{
Two-Dimensional Front Dynamics and Spatial Solitons in a Nonlinear Optical System
}

\author{
M. Pesch and W. Lange \\ Institut für Angewandte Physik, Westfälische Wilhelms-Universität Münster, Corrensstr. 2/4, D-48149 Münster, Germany \\ D. Gomila \\ Unidad de Física Interdisciplinar (CSIC-UIB), Campus Universitat Illes Balears, E-07071 Palma de Mallorca, Spain \\ T. Ackemann, ${ }^{*}$ W. J. Firth, and G.-L. Oppo \\ SUPA and Department of Physics, University of Strathclyde, Glasgow G4 ONG, Scotland, United Kingdom
}

(Received 4 May 2007; published 12 October 2007)

\begin{abstract}
Two-dimensional fronts and coarsening dynamics with a $t^{1 / 2}$ power law are analyzed experimentally and theoretically in a nonlinear optical system of a sodium vapor cell with single-mirror feedback. Modifications of the $t^{1 / 2}$ power law are observed in the vicinity of a modulational instability leading to the formation of spatial solitons of different sizes. The experimental and numerical observations give direct evidence for the locking of fronts as the mechanism of soliton formation. A phenomenological equation for the dynamics of the domain radius explains the observed behavior.
\end{abstract}

DOI: 10.1103/PhysRevLett.99.153902

The nucleation problem in systems with two equivalent or nearly equivalent phases has attracted a lot of attention in equilibrium and, more recently, nonequilibrium physics (see, e.g., [1]). Much of the interest in this problem is related to the formation of self-organized localized states (dissipative solitons). In two or more spatial dimensions the dynamics of a nucleation bubble is governed by curvature effects and possibly a "pressure force" if the two states are not exactly equivalent. In systems with a nonconserved order parameter, the resulting coarsening dynamics was shown experimentally and theoretically to follow a $t^{1 / 2}$ power law due to curvature-driven dynamics [2-4].

Nonequilibrium nonlinear optical systems are characterized by diffractive spatial coupling and typically a strong nonvariational character [5-9]. Although-contrary to first reports [8,9]—nonlinear optical systems should also present an asymptotic $t^{1 / 2}$ power law [3], there has been, so far, no experimental demonstration of this scaling.

Experimental investigations of moving fronts in optics are rare [10-12] and quantitative investigations of front dynamics are often confined to quasi-1D configurations [12]. We address two-dimensional front dynamics experimentally in a nonlinear feedback system. We demonstrate the $t^{1 / 2}$ growth law for coarsening dynamics between two equivalent homogeneous states as well as its modifications due to an imperfect pitchfork bifurcation and to incipient modulational instabilities of the background. Spatial soliton formation due to locking of the fronts $[1,6,13,14]$ in the vicinity of a pitchfork bifurcation is also observed. The interplay between front dynamics, soliton formation, and background modulational instability is demonstrated via a phenomenological eikonal equation capable of reproducing the experimental scenario. In particular, below the threshold for the occurrence of solitons, we demonstrate a slowing down of the dynamics at precursors of the
PACS numbers: 42.65.Tg, 05.45.Yv, 42.65.Sf, 47.54.-r

solitonic states. These are the localized analogue of pattern precursors in dissipative systems $[15,16]$.

The experiment is based on the well-known singlemirror feedback scheme [17] (Fig. 1). A linearly polarized collimated laser beam is injected into a heated cell containing sodium vapor as nonlinear medium. The light field transmitted by the medium propagates towards a plane mirror at a distance $d$ and is then fed back into the medium. As a modification of the standard scheme, a $\lambda / 8$ retardation plate is placed in the feedback loop to induce a partial exchange between the circularly polarized components of the light field $[10,18]$. The nonlinear optical properties of the vapor are provided by optical pumping of the $D_{1}$ transition (see [19] and references therein). An intensity difference between the circularly polarized components of

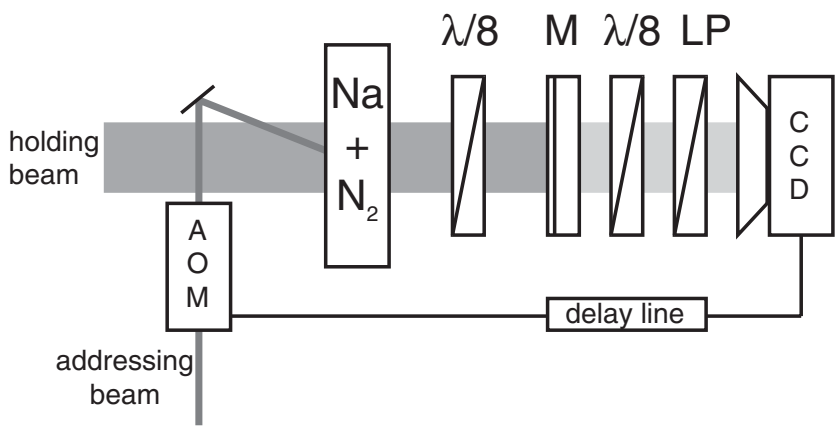

FIG. 1. Schematic experimental setup. $\lambda / 8$ retardation plate $(\lambda / 8)$, mirror $(\mathrm{M}, R \approx 0.99)$, linear polarizer (LP), CCD camera (integration time $1 \mu \mathrm{s}$ ), Acousto-optic modulator (AOM). The principal axes of the two $\lambda / 8$-plate are perpendicular to each other so that the polarization state of the light field after transmission through the cell is reestablished before detection. An "analyzer" LP in a rotary stage is used to measure the polarization of the light field and is typically adjusted to suppress the background state. 
the light field produces a population difference between the Zeeman substates of the ground state, an orientation, which determines its optical properties.

This system exhibits a symmetry-breaking bifurcation leading to two homogeneous states of the orientation when the slow axis of the retardation plate is aligned with the input polarization $[10,18]$. These states differ in the sign of both the orientation and the polarization rotation angle of the light field transmitted by the vapor. If the retardation plate is rotated by a small angle $\rho$ with respect to the input polarization, a perturbed pitchfork bifurcation is observed $[10,18]$. Since pattern formation and solitons occur at much higher input powers $[10,14]$, there is a broad range of input powers where the two orientation states are homogeneous (apart from small amplitude variations due to the Gaussian profile of the holding beam; radius $w_{0}=1.9 \mathrm{~mm}$ at the $1 / e^{2}$-point of intensity).

In this range, the system is prepared in a state with negative polarization rotation and where the unstructured states are nearly equivalent $\left(\rho \approx 0^{\circ}\right)$. Then the $\sigma_{+}$polarized addressing beam is switched on by means of an acousto-optic modulator (AOM). It is positioned at the center of the holding beam and has a radius of $\approx 1.5 \mathrm{~mm}$. Optical pumping locally creates a transition from the state with negative polarization rotation to the state with positive polarization rotation. A domain of one of the bistable unstructured states embedded into a background of the other state is thus created. If the addressing beam is switched off, coarsening dynamics sets in. The temporal evolution is analyzed by means of a video sampling technique [20]. The dynamic sequence is multiply repeated, and the point in time where the CCD camera is exposed is delayed in incremental steps (see Fig. 1). The insets in Fig. 2 show some sample frames of the time sequence [21]. Immediately after the addressing beam is turned off $(t=0)$, the domain starts to contract preserving its circular shape. The circular domain shrinks to zero in a time period of $4 \mathrm{~ms}$ when the initial background state is recovered.

In the presence of a weak symmetry breaking, the dynamics of a circular domain of radius $R$ is governed by $[2,22]$

$$
\frac{d R}{d t}=-\frac{\gamma_{c}}{R}+\gamma_{i}
$$

The first term describes curvature-driven dynamics, while the second term describes the "pressure-driven" motion of domain boundaries induced by the imperfection of the symmetry-breaking bifurcation. For $\rho=0=\gamma_{i}$ the dynamics is given by $R(t)=\left[R_{0}^{2}-2 \gamma_{c} t\right]^{1 / 2}$, i.e., a $t^{1 / 2}$ power law. The experimental shrinking of a circular domain shown in Fig. 2 is described very well by curvaturedriven dynamics. This is also confirmed by numerical simulations of a microscopic model of the system [19] with the inclusion of the action of the $\lambda / 8$-plate [10]. Numerical data obtained from this model agree very well with the experimental measurements (see the open circles

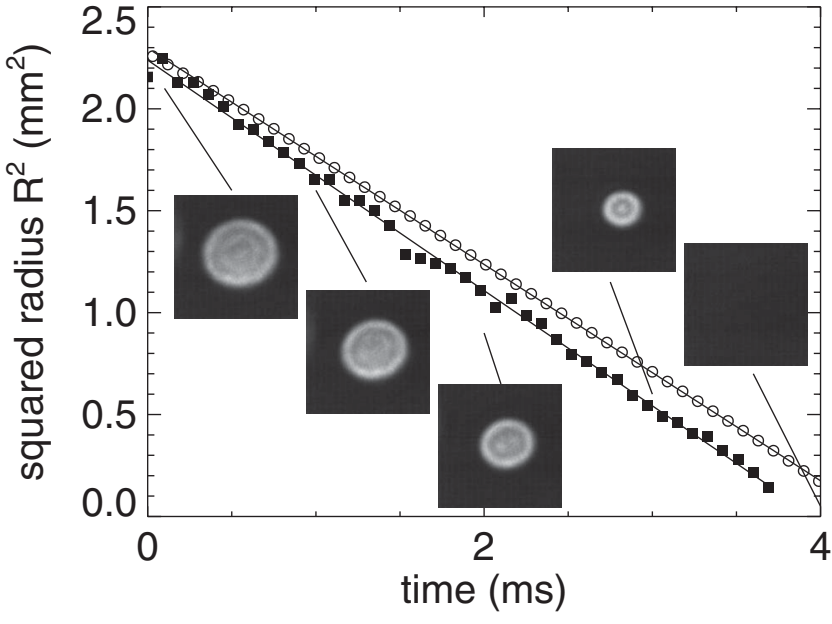

FIG. 2. The squared radius of a contracting circular domain versus time. Solid squares (open circles) denote experimental (numerical) measurements with a straight line linear fit. The insets are snapshots of the beam shape in a gray scale ("bright" denoting high intensity). The background state has been suppressed by a linear polarizer. Experimental parameters: mirror distance $d=112 \mathrm{~mm}$; buffer gas pressure $p_{N_{2}}=300 \mathrm{mbar}$; cell temperature $T=346.0^{\circ} \mathrm{C}$, laser detuning $\Delta=15.8 \mathrm{GHz}$, laser power $P_{\text {in }}=90 \mathrm{~mW}$, laser beam radius $w_{0}=1.89 \mathrm{~mm}$, rotation of the slow axis of the $\lambda / 8$ plate with respect to input polarization $\Phi=0^{\circ} 0^{\prime}$. Numerical parameters: $d=120 \mathrm{~mm}$, ground state relaxation rate $\gamma=200 \mathrm{~s}^{-1}$, relaxation rate of optical coherences $\Gamma_{2}=9.94 \times 10^{9} \mathrm{~s}^{-1}, \Delta=11.9 \mathrm{GHz}$, diffusion constant $D=255 \mathrm{~mm}^{2} \mathrm{~s}^{-1}$, particle density $N=4.65 \times 10^{19} \mathrm{~m}^{-3}$, length of heated zone $L=15 \mathrm{~mm}$, mirror reflectivity $R=$ $99.5 \%$, pump rate $P_{0}=50 \times 10^{3} \mathrm{~s}^{-1}, \Phi=0^{\circ} 0^{\prime}$.

in Fig. 2). Since the amplitude of the homogeneous orientation states has a weak dependence on the input power [10], the background states are essentially flat and the Gaussian shape of the holding beam can be neglected.

A variation of the rotation angle of the wave plate $\rho$ induces an imperfection in the pitchfork bifurcation with significant effects on the dynamics. By measuring contraction rates at different angles $\rho$ [23], we have determined the dependence of the coefficients $\gamma_{i}$ and $\gamma_{c}$ on the angle $\rho$ both experimentally and numerically (see Fig. 3 ). If the domain is disfavored $(\rho>0), \gamma_{i}$ is negative and the domain contracts faster. The case of equivalent states is recovered for $\rho \approx 0\left(\gamma_{i} \approx 0\right)$, in agreement with the theory. For negative $\rho, \gamma_{i}$ is positive and Eq. (1) predicts a critical radius $R_{\text {crit }}=\gamma_{c} / \gamma_{i}$ of an unstable nucleation droplet. For $R>R_{\text {crit }}\left(R<R_{\text {crit }}\right)$ growth (contraction) of a circular domain is expected. This corresponds nicely to the experimental and numerical observations. Finally, the inset of Fig. 3 shows that $\gamma_{c}$ moderately increases with increasing rotation angles. This tendency is also present in the simulation although considerably less pronounced.

The dependence of the coefficient $\gamma_{c}$ on the input power is plotted in Fig. 4. If the input power of the holding beam is increased, the dynamics is very well described in the framework of curvature-driven motion following Eq. (1) 


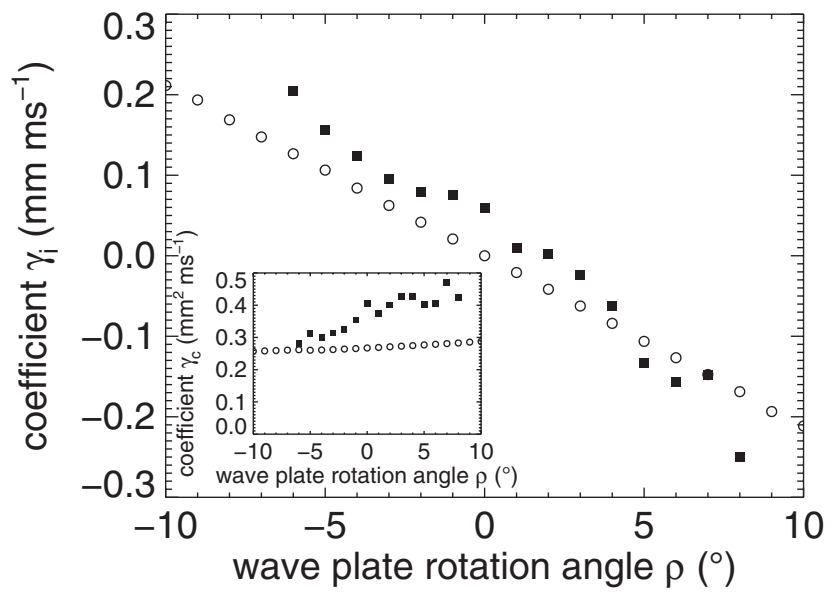

FIG. 3. Coefficients $\gamma_{i}$ and $\gamma_{c}$ versus the wave plate rotation angle $\rho$. Solid squares are experimental results, open circles have been obtained by numerical simulations.

up to an input power of approximately $150 \mathrm{~mW}$ [23]. Here, the data show scatter in the low power range, with overall a weak decrease of $\gamma_{c}$ with increasing $P_{\text {in }}$ as confirmed by the numerical simulations. Such weak dependence of $\gamma_{c}$ on $P_{\text {in }}$ provides further evidence that the intensity variation of the Gaussian holding beam does not influence the coarsening dynamics significantly.

Above an input power of $\approx 150 \mathrm{~mW}$, there is a qualitative change in the dynamical behavior of the domains [23]. Figure 5 shows the temporal evolution of a domain at high input powers in an overlay of three sampling sequences at equal values of the parameters. While the monotonic decrease of the domain radius persists, the contraction curve shows a large amount of modulations and two main plateaus. The insets of Fig. 5 show images of the domains at the radii of the plateaus [21]. These structures display

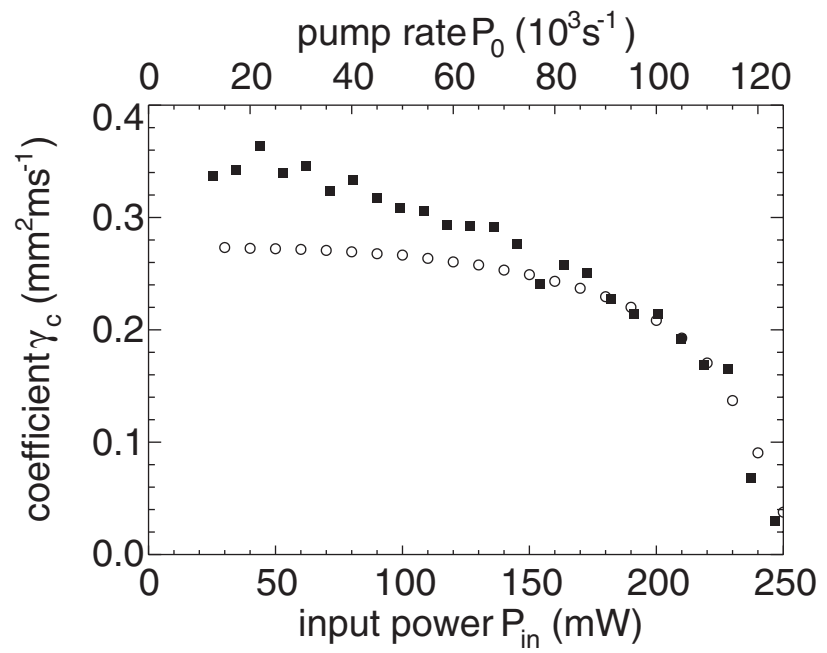

FIG. 4. Coefficient $\gamma_{c}$ as a function of the input power (experiment) and pump rate (simulations). Here, experimentally, $T=354.7^{\circ} \mathrm{C}, \Delta=17.5 \mathrm{GHz}$. pronounced radial oscillations due to an incipient modulational instability at higher input powers. With increasing input power, larger spatial oscillations and a further slowing-down are observed until spatial solitons become stable. Hence the states at which the dynamics nearly stops are interpreted as precursors of the stable solitons due to locking of the fronts [14].

As a result of the ring interactions a discrete family of solitons is observed in parameter regions where front velocities are low and locking is promoted by the appearance of pronounced spatial oscillations. The regions of soliton existence from the experiment and from a numerical Newton method are presented in Figs. 6(a) and 6(b). In contrast to one-dimensional systems where the curvaturedriven dynamics is absent, the thresholds for the existence of each soliton are shifted towards negative $\rho$ and positive $\gamma_{i}$ where the imperfection of the pitchfork bifurcation tends to compensate for the curvature-driven contraction. Above the cusp thresholds, broad regions of soliton existence with large overlap and multistability are observed.

The interaction and locking of oscillatory tails of domain boundaries has often been considered to warrant the stabilization of domains in one-dimensional systems [1,6]. Although the extension of such a locking phenomenon to two spatial dimensions is nontrivial, its main features can be captured phenomenologically by a modified equation for the radius dynamics. The interaction of two fronts separated by a distance $d$ and due to their oscillatory tails leads to a force of the type $f(d)=g \cos (\beta d) e^{-\alpha d}$ [24]. It is therefore reasonable to model the evolution of the radius of a circular domain wall using the following ansatz:

$$
\frac{d R}{d t}=-\frac{\gamma_{c}}{R}+\gamma_{i}+g \cos (\beta 2 R) e^{-\alpha 2 R},
$$

where $\alpha$ and $\beta$ are calculated from the real and imaginary parts of the eigenvalues of the most underdamped spatial modulations of the homogeneous solutions. $\beta$ corresponds

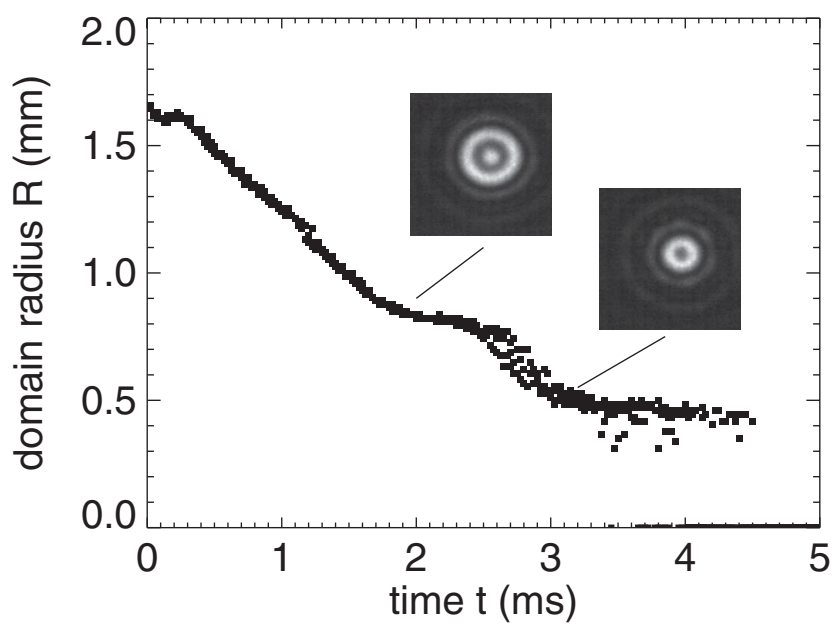

FIG. 5. Contraction of a circular domain close to the locking region $\left(P_{\text {in }}=190 \mathrm{~mW}\right)$. Insets show images of the domain at the plateaus of the contraction curve. 

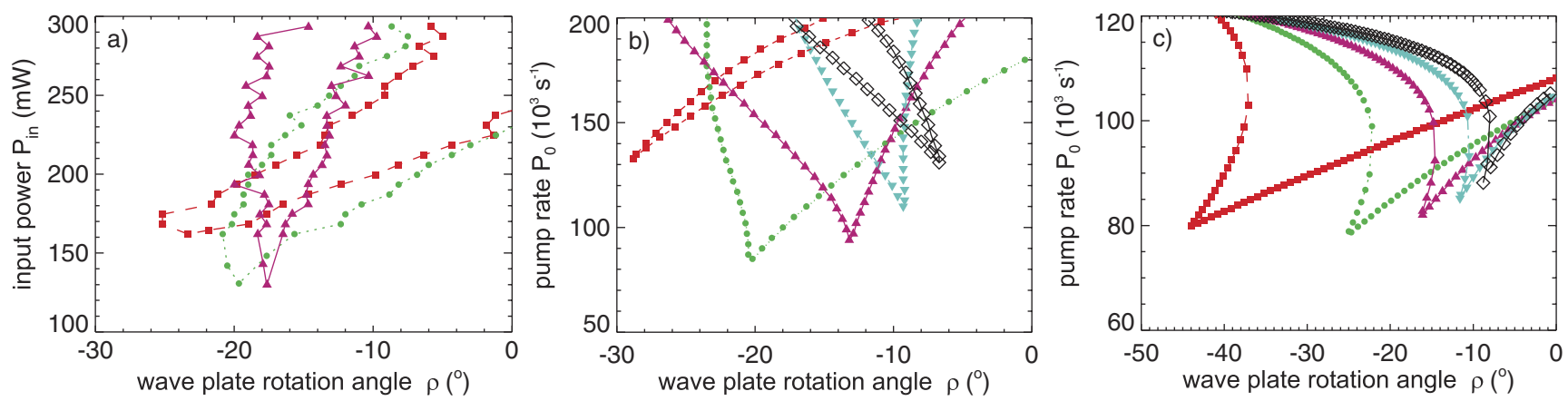

FIG. 6 (color online). Regions of existence of dissipative solitons of different orders [from first to fifth order: squares (red), circles (green), up-triangles (magenta), down-triangles (cyan), diamonds (black)]. (a) Experiment; (b) Newton method; (c) analytic solution of Eq. (2). Here in (a) $T=360.2^{\circ} \mathrm{C}$ and $\Delta=16.3 \mathrm{GHz}$. In (b) $w_{0}=1.89 \mathrm{~mm}$.

to the wave number of the modulations, $\alpha$ is the decay rate of the modulations and vanishes at the modulational instability point. For simplicity we assume a linear dependence of $\alpha$ with the pump $P_{0}: \alpha \propto\left(P / P_{c}-1\right)$. For $\gamma_{c}$ we take a linear dependence with $P_{0}$, which is a rough approximation of the experimental data shown in Fig. 4. $\gamma_{i}$ is linear with $\rho$ (see Fig. 3). This simple eikonal equation qualitatively reproduces the bifurcation structure and region of existence in the parameter space of the dissipative solitons [see Fig. 6(c)]. Strictly speaking, such equations should be valid only for large order localized structures where the radius is still much larger than the front width. However, the qualitative agreement is acceptable even for the second order dissipative solitons. An important difference of this discrete family of dissipative solitons with that of 1D systems is that for small asymmetries higher order dissipative solitons have lower thresholds than the fundamental one. In fact, the locking due to the oscillatory tails occurs now around the unstable nucleation radius $R_{\text {crit }}$. When the pump is increased, the most favored localized solution is that one with the radius closest to $R_{\text {crit }}$. The unstable nucleation radius $R_{\text {crit }}=\gamma_{c} / \gamma_{i}$ is typically quite large, although it decreases with increasing asymmetry $\left(\gamma_{i}\right)$. The order of the first dissipative soliton to appear decreases, then, with increasing $|\rho|$ [see Fig. 6(b) and 6(c)].

Summarizing, we have demonstrated and analyzed different regimes of growth laws in a 2D optical system with a pitchfork bifurcation and approaching a modulational instability. The measurements and analysis provide direct evidence for the stabilization mechanism for solitons in these systems proposed in [14].

We acknowledge travel support from the Royal Society and the Faculty of Science of the University of Strathclyde. We thank P. Coullet and C. Denz for fruitful discussions.

*thorsten.ackemann@strath.ac.uk

[1] P. Coullet, Int. J. Bifurcation Chaos Appl. Sci. Eng. 12, 2445 (2002).
[2] S. M. Allen and J. W. Cahn, Acta Metall. 27, 1085 (1979). For reviews see J. D. Gunton, M. San Miguel, and P. Sahni, in Phase Transitions and Critical Phenomena, edited by C. Domb and J. Lebowitz (Academic, New York, 1983), Vol. 8, p. 269; A. Bray, Adv. Phys. 43, 357 (1994).

[3] D. Gomila et al., Phys. Rev. Lett. 87, 194101 (2001); IEEE J. Quantum Electron. 39, 238 (2003).

[4] B. Marts, K. Martinez, and A. L. Lin, Phys. Rev. E 70, 056223 (2004).

[5] N. Akhmediev and A. Ankiewicz, Dissipative Solitons, Lecture Notes in Physics Vol. 661 (Springer, Berlin, 2005).

[6] G.-L. Oppo, A. J. Scroggie, and W. Firth, J. Opt. B 1, 133 (1999); G.-L. Oppo et al., J. Mod. Opt. 47, 2005 (2000); Phys. Rev. E 63, 066209 (2001).

[7] R. Gallego, M. San Miguel, and R. Toral, Phys. Rev. E 61, 2241 (2000).

[8] M. Tlidi, P. Mandel, and R. Lefever, Phys. Rev. Lett. 81, 979 (1998); M. Tlidi et al., Opt. Lett. 25, 487 (2000); M. Tlidi and P. Mandel, Europhys. Lett. 44, 449 (1998).

[9] M. Le Berre, E. Ressayre, and A. Tallet, J. Opt. B 2, 347 (2000).

[10] E. Große Westhoff et al., J. Opt. B 2, 386 (2000).

[11] Ye. Larionova et al., Phys. Rev. A 69, 033803 (2004).

[12] A. Esteban-Martin et al., Phys. Rev. Lett. 94, 223903 (2005).

[13] V. B. Taranenko et al., Phys. Rev. A 56, 1582 (1997).

[14] M. Pesch et al., Phys. Rev. Lett.95, 143906 (2005).

[15] G. Agez et al., Phys. Rev. A 66, 063805 (2002).

[16] I. Rehberg et al., Phys. Rev. Lett. 67, 596 (1991).

[17] G. D'Alessandro and W. J. Firth, Phys. Rev. Lett. 66, 2597 (1991).

[18] T. Yabuzaki et al., Phys. Rev. A 29, 1964 (1984).

[19] A. Aumann et al., Phys. Rev. E 66, 046220 (2002).

[20] M. Möller et al., Chaos Solitons Fractals 10, 675 (1999).

[21] The whole sequence is available as an online video file.

[22] D. Gomila et al., J. Opt. B 6, S265 (2004).

[23] See EPAPS Document No. E-PRLTAO-99-025742 for supplementary material and animated versions of the dynamics and snapshots in Figs. 2 and 5. For more information on EPAPS, see http://www.aip.org/pubservs/ epaps.html.

[24] P. Coullet, C. Elphick, and D. Repaux, Phys. Rev. Lett. 58, 431 (1987). 